\title{
Contact between the components of a knee prosthesis: numerical and experimental study
}

\section{Contatto tra i componenti di una protesi di ginocchio: studio numerico e sperimentale}

\author{
D. Cerniglia, T. Ingrassia, L. D’Acquisto, M. Saporito \\ Dipartimento di Ingegneria Chimica, Gestionale, Informatica, Meccanica Università degli Studi di Palermo - viale delle Scienze, \\ 90128 Palermo (Italy) \\ donatella.cerniglia@unipa.it
}

D. Tumino

Facoltà di Ingegneria, Architettura e delle Scienze Motorie Università degli Studi di Enna Kore - Cittadella Universitaria, 94100 Enna (Italy)

\begin{abstract}
The aim of this work is the analysis of the contact area in a knee prosthesis using two different approaches. In particular, the interface between the femoral component and the polyethylene insert has been studied both numerically and experimentally. The interest in studying the contact area is related to the fact that the wear of the polyethylene insert, due to the high contact pressures, represents one of the major causes of failure of the total knee prosthesis. The possibility to evaluate the contact area at different loads and mutual position between femur and tibia is, therefore, of fundamental importance to study the service life of a prosthesis and to improve its performance. The finite element numerical approach has required the acquisition, through reverse engineering, and CAD modelling of the prosthetic components. Then the FEM simulations have been developed considering two different load conditions. In order to compare the calculated data, the same load configurations have been used for experimental tests based on ultrasonic method. In this case, some preliminary tests were required to calibrate the system depending on the particular characteristics of materials, geometries and surface finish of the prosthesis.

The results show a good correlation between the data obtained with the two different approaches and, consequently, a good level of reliability of the procedures developed for the numerical and experimental evaluation of the contact area. The numerical procedure can be used to determine the area for different angles and loads, but especially in the design phase. The ultrasonic technique can be used to validate the numerical data.
\end{abstract}

SOMMARIO. Scopo del presente lavoro è lo studio dell'area di contatto in una protesi di ginocchio utilizzando due differenti approcci. In particolare è stata studiata l'interfaccia fra la componente femorale e l'inserto in polietilene sia numericamente che sperimentalmente. L'interesse allo studio dell'area di contatto è legato al fatto che, molto spesso, l'usura dell'inserto in polietilene, dovuta alle elevate pressioni di contatto, rappresenta una delle principali cause di fallimento delle protesi totali di ginocchio. La possibilità di valutare l'area di contatto al variare dei carichi e della posizione reciproca fra femore e tibia è, quindi, di fondamentale importanza per lo studio della vita utile di una protesi e per cercare di migliorarne le prestazioni. L'approccio numerico agli 
elementi finiti ha richiesto l'acquisizione, tramite tecniche di reverse engineering, e la modellazione CAD delle varie componenti della protesi. Successivamente sono state messe a punto le simulazioni FEM considerando due diverse condizioni di carico. Al fine di rendere paragonabili i dati calcolati, le stesse configurazioni di carico sono state utilizzate per le prove sperimentali tramite tecnica ultrasonora. In questo caso, è stato necessario effettuare alcune prove preliminari per tarare il sistema in dipendenza delle particolari caratteristiche dei materiali, delle geometrie e delle finiture superficiali della protesi.

I risultati ottenuti mostrano un buon grado di correlazione fra i dati rilevati seguendo i due differenti approcci e, di conseguenza, un buon livello di affidabilità delle procedure messe a punto per la determinazione numerica e sperimentale dell'area di contatto. La procedura numerica può essere usata per determinare l'area per diversi angoli e per diversi carichi, ma soprattutto in fase di progettazione. La tecnica ad ultrasuoni può essere usata per validare i dati numerici.

Parole Chiave. Protesi di ginocchio; Ultrasuoni; Reverse engineering; Analisi FEM.

\section{INTRODUZIONE}

T 'articolazione del ginocchio umano è una complicata struttura che deve soddisfare contemporaneamente due esigenze apparentemente contrastanti: consentire un'adeguata mobilità per permettere la normale locomozione e possedere la necessaria stabilità per dare un corretto supporto ai compiti motori da eseguire. A causa di traumi e patologie, la sua funzionalità può essere compromessa a tal punto da rendere indispensabile intervenire chirurgicamente mediante ricostruzione parziale o totale dell'articolazione stessa. Gli interventi correlati vanno sotto il nome di artroplastica totale o parziale di ginocchio, per cui le parti danneggiate dell'articolazione vengono sostituite con protesi, grazie alle quali l'articolazione riacquista la normale funzionalità.

La vita utile di una protesi è difficile da calcolare, ma grazie ai continui progressi tecnologici i pazienti possono contare su impianti protesici di durata sempre maggiore. Proprio su questa linea guida, in questo lavoro si è studiato uno dei principali problemi che portano al fallimento dell'impianto protesico: l'usura dell'inserto in polietilene. Geometrie articolari a bassa conformità riducono lo stress trasmesso alle interfacce di fissazione ma garantiscono ridotte aree di contatto predisponendo all'usura precoce ed allo slaminamento del cuscinetto in polietilene, in quanto il carico si ripartisce in un area di dimensioni inferiore [1]. Lo stress di contatto rilevato sperimentalmente sulle superfici di adesione del polietilene risulta infatti inversamente proporzionale all'estensione della superficie di congruenza tra i capi articolari giustapposti [2]. Di contro, però, all'aumentare del grado di conformità si riduce la mobilità e si incrementano gli sforzi torsionali e di taglio.

Le tecniche utilizzabili per la valutazione dell'area di contatto possono essere sia di tipo numerico [3] che sperimentale. La letteratura riporta alcuni risultati relativi all'utilizzo di tecniche sperimentali quali tecniche ad inchiostro, joint-casting, stereofotogrammetria, pellicole pressosensibili $[4,5]$, tecniche ultrasoniche. In generale, però, le tecniche sperimentali che interpongono un mezzo di riscontro tra le superfici accoppiate, possono variare lo stato del contatto fra le interfacce, falsando in modo indiretto i risultati ottenuti. L'applicazione di metodi di indagine ultrasonora allo studio di problemi di contatto si basa essenzialmente sulla nota tecnica pulse-echo, ampiamente utilizzata nei controlli non-distruttivi in campo industriale [6-12].

L'obbiettivo del presente lavoro è quello di caratterizzare il contatto tra le superfici di accoppiamento di un impianto protesico attraverso due diversi approcci, numerico e sperimentale, e confrontarne i risultati. Le prove numeriche sono state eseguite attraverso analisi agli elementi finiti mentre, l'indagine sperimentale è stata sviluppata impiegando la tecnica ultrasonora. Le prove sono state effettuate per due differenti livelli di carico associati a due diversi angoli di inclinazione.

\section{GINOCCHIO ED ENDOPROTESI ORTOPEDICHE}

$\mathrm{L}$ 'articolazione del ginocchio è sicuramente una delle più complesse, sia dal punto di vista anatomico che funzionale. Pur disponendo di un'elevata mobilità tra femore e tibia durante la marcia, ha un'ottima stabilità durante le fasi di carico statico dovuta alla presenza di una complessa struttura legamentosa. La flessione del ginocchio non è un semplice movimento di rotolamento della tibia rispetto al femore, bensì un rotolamento seguito da uno scivolamento. 
Questo permette un ampio movimento di flesso-estensione durante il quale il ginocchio può raggiungere una flessione massima di circa $160^{\circ}$; valore di molto superiore a quello che si potrebbe ottenere con un rotolamento puro, a parità di spostamento del punto di contatto sulle superfici [1].

Le endoprotesi ortopediche sono dei sistemi impiantabili permanentemente all'interno del corpo umano dove svolgono il loro ruolo in diretto contatto con i tessuti dell'organismo. La protesi di ginocchio permette a pazienti che hanno subito traumi o affetti da grave artrosi del ginocchio il recupero di un'elevata qualità di vita, con un buon recupero funzionale e la riduzione della sintomatologia dolorosa. La chirurgia protesica del ginocchio si è evoluta notevolmente negli ultimi anni affrontando l'esigenza dei pazienti di avere una precoce rieducazione ed una minore invasività dell'intervento. Gli interventi di sostituzione protesica di ginocchio possono essere classificati in tre tipologie:

1) totale: prevede di intervenire su entrambe le componenti articolari, femorale e tibiale;

2) monocompartimentale: quando l'artrosi interessa solo la parte esterna o interna del ginocchio;

3) revisione o riprotesizzazione: prevede la sostituzione di un dispositivo precedentemente impiantato.

La protesi totale di ginocchio è costituita da alcuni elementi che sostituiscono funzionalmente l'articolazione fisiologica. La componente tibiale è costituita da un piatto di supporto metallico ed un inserto di polietilene; la componente femorale è in metallo e riproduce la forma anatomica dei condili. La qualità dell'osso, la morfologia femorale e tibiale, l'età del paziente indirizzano la scelta del modello della protesi, della taglia e del mezzo di fissazione.

\section{Protesi "Multigen Plus" e condizioni di carico}

Le prove sperimentali sono state eseguite sulla protesi totale di ginocchio Multigen Plus, fornita dalla Lima-Lto [13]. Si tratta di una protesi totale costituita da tre componenti accoppiati: quella femorale, quella tibiale ed un inserto in polietilene posizionato tra le due (Fig. 1).

La componente femorale è in CoCrMo con rivestimento interno in titanio poroso. La finitura interna conferisce alla protesi una macrorugosità superficiale molto accentuata (Fig. 2), creata per migliorare le caratteristiche di ostointegrazione della protesi. La particolare finitura superficiale è stata oggetto di studio nella fase preliminare delle prove sperimentali, perché risulta responsabile di fenomeni di attenuazione del segnale ultrasonoro.

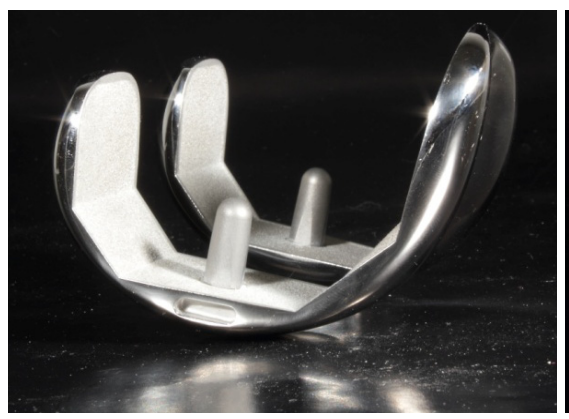

(a)

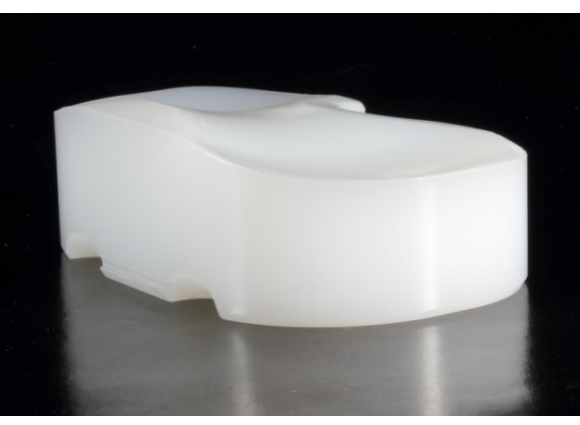

(b)

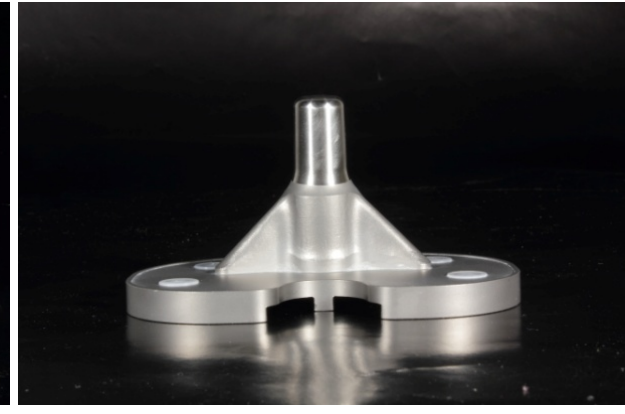

(c)

Figura 1: Protesi totale di ginocchio: a) componente femorale, b) inserto in UHMWPE, c) componente tibiale. Figure 1: Knee prosthesis: a) femoral component, b) UHMWPE insert, c) tibial component.

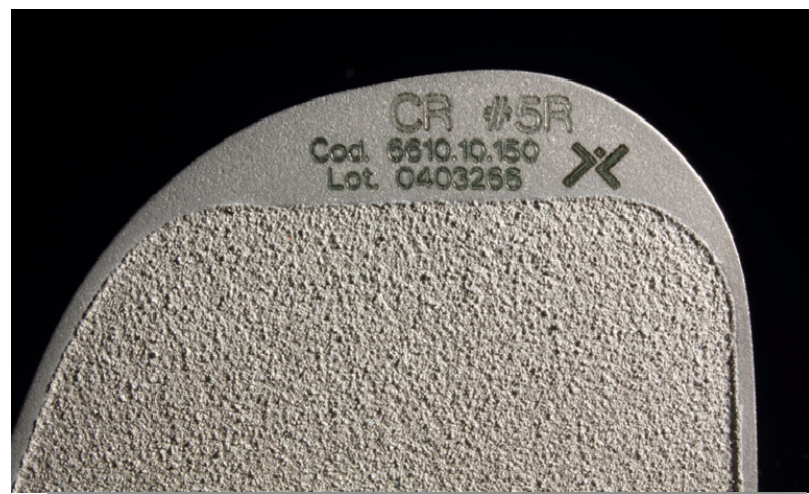

Figura 2: Finitura superficiale interna in titanio poroso della componente femorale.

Figure 2: Porous titanium surface roughness of the femoral component. 
L'inserto è in polietilene ad elevatissimo peso molecolare (UHMWPE), materiale biocompatibile che da oltre trent'anni viene utilizzato nella fabbricazione di impianti protesici, grazie alle sue caratteristiche vantaggiose: basso coefficiente d'attrito, elevata resistenza all'usura (massima tra i polimeri), elevata resistenza all'urto ed elevata stabilità chimica. Come tutti i materiali viscoelastici, l'UHMWPE è soggetto a deformazione per scorrimento viscoso se sottoposto a carico costante. Dal punto di vista meccanico l'UHMWPE presenta le caratteristiche tipiche di molti polimeri: basso sforzo di rottura $(35 \mathrm{MPa})$, basso limite di snervamento $(21 \mathrm{MPa})$, elevato allungamento a rottura $(350 \%)$, modulo elastico pari a $0.8-1.5 \mathrm{GPa}$.

I principali fattori di degradazione sono la sensibilità all'ossidazione, che conduce a una diminuzione del peso molecolare, e allo stress cracking (creazione all'interno di microvuoti); la combinazione di questi due processi riduce, inoltre, la resistenza a fatica, creando la possibilità di frattura fragile. Nel caso delle protesi d'anca e di ginocchio si verificano fenomeni di usura dell'UHMWPE, con una velocità di $0.1-0.6 \mathrm{~mm} /$ anno. Studi istologici di tessuti periprotesici mostrano la presenza di detriti e di particelle di polietilene rilasciati da fenomeni di usura [14]. Le dimensioni di queste particelle sono ampiamente variabili e possono provocare una reazione infiammatoria che, nei casi limite, può essere responsabile della necrosi dell'osso. La minimizzazione dell'usura è una specifica di progetto assolutamente necessaria per il buon funzionamento dell'impianto protesico.

Il piatto tibiale fisso è realizzato in Ti $6 \mathrm{Al} 4 \mathrm{~V}$ cementato mediante uno speciale rivestimento, che ne aumenta la rigidezza dell'impianto e ne migliora la stabilità in modo da ridurre l'usura nella zona di carico. E' una lega molto vantaggiosa per gli impianti protesici grazie al suo basso modulo elastico, alla buona resistenza a trazione e compressione, alla buona resistenza a fatica e all'ottima biocompatibilità. L'ancoraggio al tessuto osseo viene eseguito mediante viti e un opportuno fittone, che ne garantisce una corretta inserzione nello stelo tibiale, evitando fenomeni di mobilizzazione che sono la principale causa di fallimento dell'impianto protesico.

Per quanto riguarda i carichi agenti nell'articolazione durante le normali condizioni di utilizzo, c'è da sottolineare come questi dipendano dalla particolare attività svolta e dal peso corporeo. Nella condizione di angolo di flesso-estensione $\theta$ pari a zero (Fig. 3), in cui non è necessaria attività muscolare e la trazione al tendine è nulla, la forza massima che femore e tibia si scambiano è pari al peso corporeo $\mathrm{P}$.
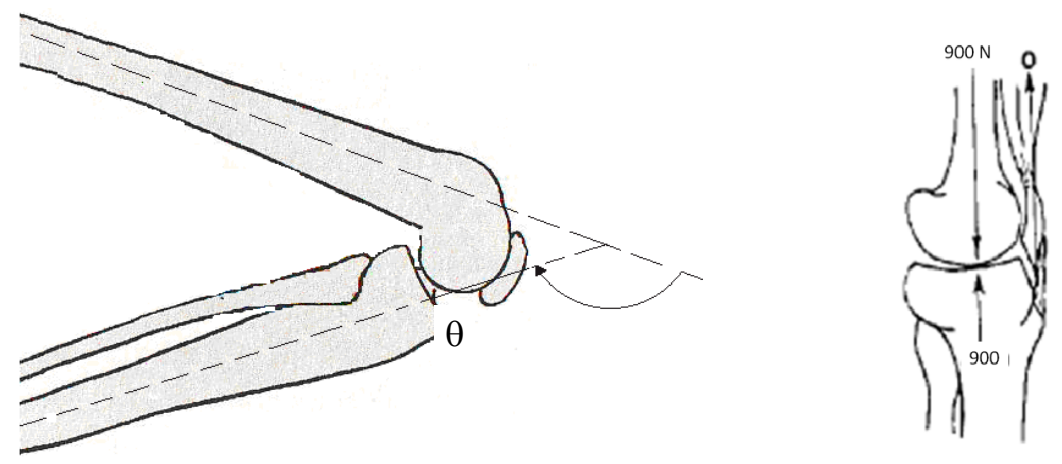

Figura 3: Convenzione per l'angolo di flesso-estensione $\theta$, compreso tra il prolungamento del segmento coscia rispetto al segmento gamba, e configurazione per $\theta$ pari a zero.

Figure 3: Angle of flexion-extension $\theta$, between the extension of the femoral axis with respect to the tibial axis, and configuration for $\theta=0^{\circ}$.

Nel caso in cui l'angolo di flesso-estensione varia, condizione che si realizza anche durante la normale deambulazione, il valore della forza agente sul ginocchio dipende da esso. Questa può essere efficacemente stimata considerando un coefficiente moltiplicatore rispetto al peso corporeo P (Tab. 1) [1].

\begin{tabular}{lcc}
\hline Attività & Intervallo di rotazione & Forze sul ginocchio \\
Passeggiare & $0-67^{\circ}$ & $3.02 \mathrm{P}$ \\
Salire le scale & $0-83^{\circ}$ & $4.25 \mathrm{P}$ \\
Scendere le scale & $0-90^{\circ}$ & $3.83 \mathrm{P}$ \\
\hline
\end{tabular}

Tabella 1: Intervalli di rotazione durante attività usuali e valori tipici di forza sul ginocchio in funzione delle diverse attività $(\mathrm{P}=$ peso corporeo).

Table 1: Angle ranges for usual activities and typical values of force on the knee ( $\mathrm{P}=$ body weight). 
In particolare, nel presente lavoro, sono state considerate due differenti condizioni di carico per la protesi. Nella prima si è studiato un angolo di flesso-estensione pari $0^{\circ}$, nella seconda pari a $30^{\circ}$. In entrambi i casi si è considerato un individuo di peso corporeo pari a $900 \mathrm{~N}$ durante una passeggiata. I carichi utilizzati sono stati di $900 \mathrm{~N}$ per l'angolo pari a $0^{\circ}$ e di 2700 $\mathrm{N}\left(\right.$ circa 3P) nella condizione di flessione a $30^{\circ}$.

\section{VALUTAZIONE NUMERICA DELL'AREA DI CONTATTO}

'approccio numerico per la valutazione dell'area di contatto fra la componente femorale e l'inserto in polietilene, si è sviluppato in tre distinte fasi: acquisizione, tramite tecnica di reverse engineering, delle forme delle componenti della protesi, modellazione ed assemblaggio dei modelli CAD, messa a punto delle simulazioni agli elementi finiti.

\section{Reverse engineering e modellazione CAD della protesi}

La forma 3D della protesi è stata acquisita utilizzando il sistema di scansione 3D COMET 5, composto da una videocamera da 11 mega-pixel, una sorgente laser, una workstation ed un software, il COMETPlus, che gestisce i dati dalla fase di scansione fino all'esportazione di un modello CAD o della nuvola di punti acquisita. Il sistema permette di acquisire all'interno del volume di lavoro variabile da un minimo di 80 ad un massimo di $1000 \mathrm{~mm}^{3}$, ha livello di accuratezza (dipendente dal volume di acquisizione) minore di $5 \mu \mathrm{m}$ e un tempo di acquisizione molto ridotto (circa $1 \mathrm{~s}$ ). La procedura di acquisizione delle singole componenti della protesi è stata la seguente. Inizialmente, le superfici da acquisire sono state opacizzate con uno spray rivelatore bianco in modo da limitare il disturbo prodotto da eventuali riflessioni o eccessivo assorbimento della luce proiettata. Successivamente si è proiettato, per mezzo della sorgente di luce laser, un pattern regolare di frange sulle superfici; il pattern di frange risultante è stato dunque elaborato in accordo con i principi ottici della tecnica Moirè [15]. Per ottenere una descrizione completa (punto a punto) delle superfici scansionate, sono state realizzate diverse acquisizioni ruotando le componenti lungo un asse verticale.

Le nuvole di punti dei modelli acquisiti utilizzando questa tecnica sono, solitamente, soggetti a rumore (Fig. 4) e, per tale motivo, è necessario filtrare i dati prima di poter disporre di dati corretti e coerenti. A tal uopo è stato utilizzato il software Geomagic Studio, grazie al quale le nuvole di punti dei modelli acquisiti sono state filtrate, opportunamente rifinite, interpolate in superfici NURBS (Fig. 4) e convertite in modelli solidi CAD.
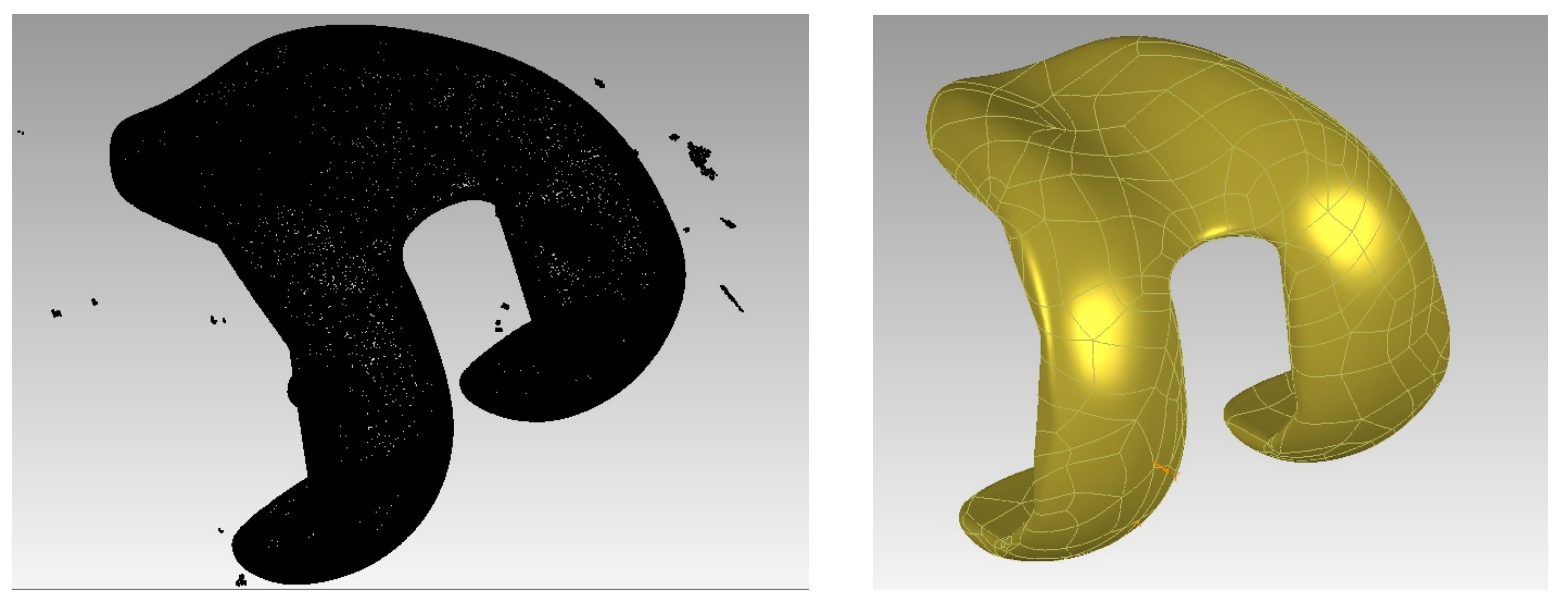

Figura 4: Componente femorale: a sinistra la nuvola di punti acquisiti, a destra il modello NURBS.

Figure 4: Femoral component: the point cloud acquired (left) and the NURBS model (right).

Attraverso il software Solid Edge i modelli CAD delle varie componenti protesiche sono stati opportunamente assemblati al fine di ottenere due modelli che riproducessero le condizioni di flesso-estensione nulla (angolo di $0^{\circ}$ ) e pari a $30^{\circ}$ (Fig. 5). L'assemblaggio dei componenti è stato eseguito in modo semiautomatico procedendo a verificare l'assenza di interferenza reciproca fra i vari pezzi attraverso l'utilizzo del modellatore Solid Edge e di un sistema di visualizzazione stereoscopica [16]. I modelli assemblati delle configurazioni a $0^{\circ}$ e $30^{\circ}$ sono stati convertiti in formato IGES per il successivo studio agli elementi finiti. 

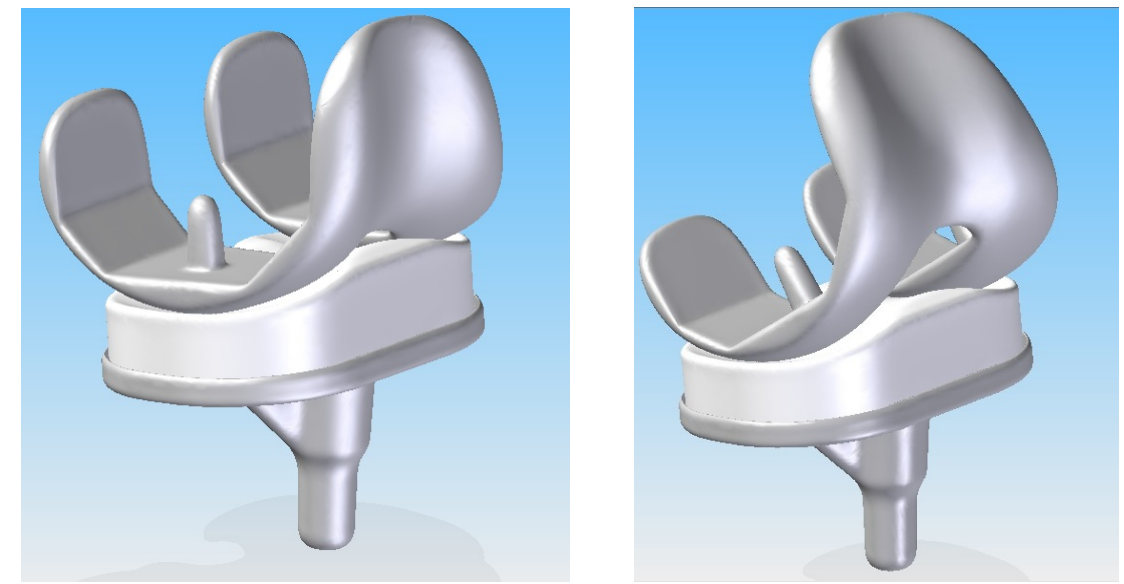

Figura 5: Modelli CAD della protesi: a sinistra la configurazione a $0^{\circ}$, a destra quella a $30^{\circ}$.

Figure 5: Knee prosthesis CAD models: $0^{\circ}$ (left) and $30^{\circ}$ (right) configuration.

\section{Analisi FEM}

I modelli assemblati sono stati importati nel software Ansys Workbench e discretizzati con elementi solidi ad otto nodi. La mesh è stata costruita utilizzando una procedura semiautomatica presente in Ansys Workbench (Adapt-automated adaptive meshing procedure) che esegue un'analisi di convergenza finalizzata alla minimizzazione della variazione dell'energia di deformazione all'interno dei singoli elementi. Tale mesh, con gli opportuni infittimenti nelle zone di contatto fra la componente femorale e l'inserto in polietilene, è mostrata in Fig. 6.

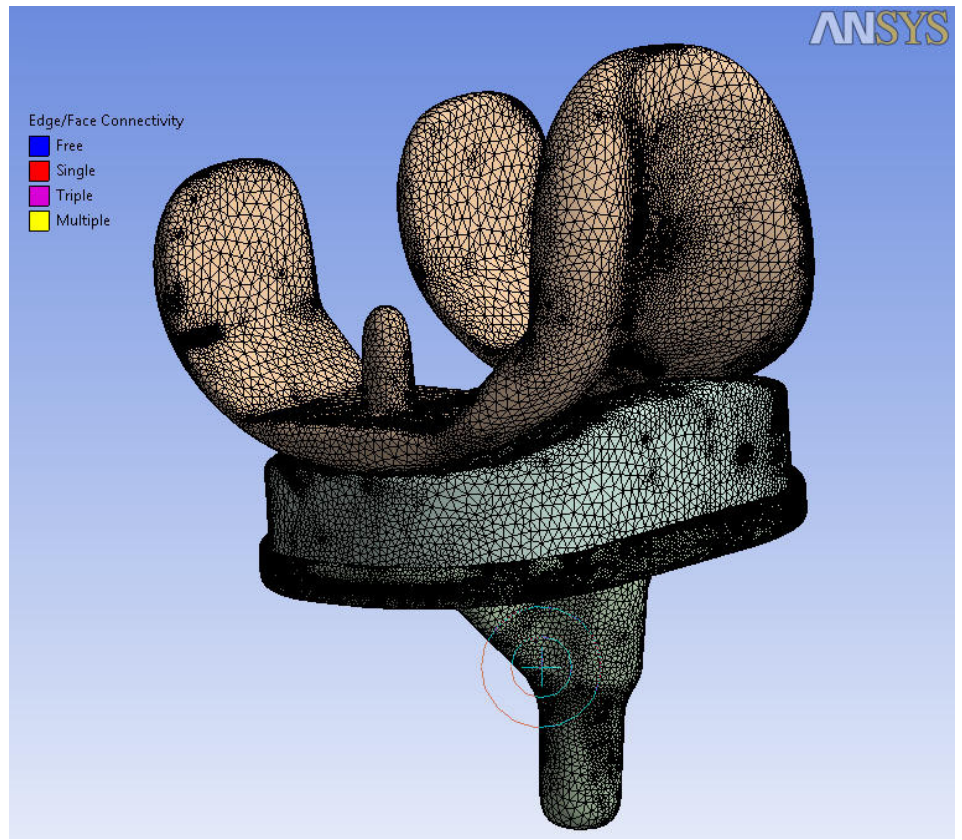

Figura 6: Mesh della protesi per la configurazione a $0^{\circ}$.

Figure 6: Knee prosthesis mesh for the $0^{\circ}$ configuration.

Successivamente sono state definite le caratteristiche dei diversi materiali impiegati e sono state impostate le condizioni di contatto fra i vari componenti protesici. In particolare, è stato utilizzato il metodo Augumented Lagrangian [17] e sono state impiegate due diverse tipologie di contatto del tipo surface-to-surface:

$\checkmark$ vincolato, per le connessioni fra l'inserto in UHMWPE ed il piatto tibiale. Utilizzando questo tipo di vincolo si ipotizza la perfetta adesione fra le facce delle regioni di contatto le quali non possono slittare né separarsi;

$\checkmark$ con attrito, per la connessione fra la componente tibiale e l'inserto in polietilene (Fig. 7). È stato scelto un coefficiente d'attrito pari a 0.01 , valore coerente con quelli riscontrati in bibliografia $[3,18]$ per tale accoppiamento di materiali. 
Tale scelta, pur essendo arbitraria, non inficia la validità dei risultati in quanto, come dimostrato in [18], il valore del coefficiente d'attrito ha un'influenza trascurabile sulla valutazione delle pressioni al contatto.

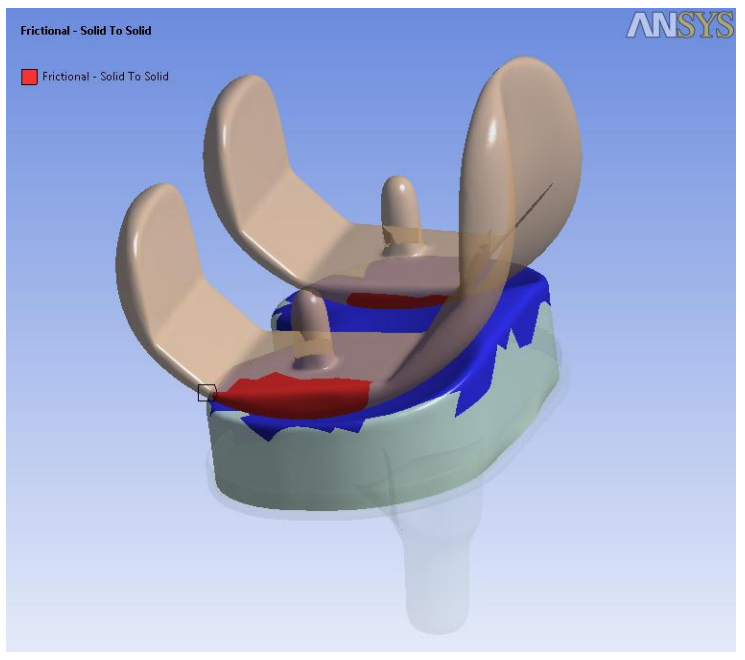

Figura 7: Definizione delle aree di contatto fra componente femorale e inserto in UHMWPE.

Figure 7: Contact area between the femoral component and the UHMWPE insert.

Infine sono state imposte le condizioni al contorno relative a carichi e vincoli, cercando di riprodurre, in modo fedele, il setup utilizzato per le prove sperimentali. Come anticipato precedentemente, sono state analizzate due configurazioni al variare dell'angolo fra femore e tibia. È stata considerata una prima configurazione con angolo pari a $0^{\circ}$ ed una seconda con angolo di $30^{\circ}$ (valore intermedio che l'articolazione raggiunge durante la passeggiata). Per entrambe le configurazioni analizzate il piatto tibiale è stato bloccato (incastro perfetto), mentre sulla componente femorale (Fig. 8) è stato imposto uno spostamento in direzione $\mathrm{x}$ positiva di modulo crescente e sono stati vincolati $\mathrm{i}$ rimanenti gradi di libertà. In particolare, lo spostamento verticale agente sulla componente femorale è stato aumentato nel tempo fino a quando il valore della forza di reazione vincolare lungo l'asse x risultasse pari a $900 \mathrm{~N}$ e $2700 \mathrm{~N}$, rispettivamente per la configurazione a $0^{\circ}$ e $30^{\circ}$.

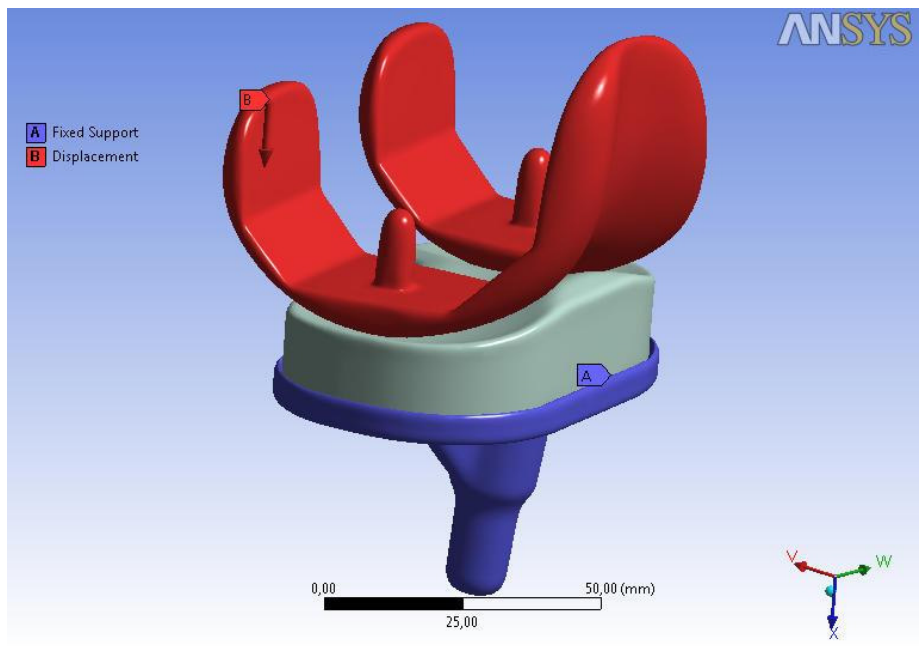

Figura 8: Condizioni al contorno: in blu il piatto tibiale bloccato, in rosso la componente femorale sulla quale è stato imposto lo spostamento verticale.

Figure 8: Boundary conditions: fixed tibial support in blue, femoral component in red where vertical displacements are applied.

Le figure seguenti mostrano le aree di contatto e la localizzazione della pressione massima all'interfaccia fra i condili della componente femorale e l'inserto in polietilene. Nel caso della configurazione a $0^{\circ}$ (Fig. 9), l'area di contatto, di forma leggermente ellittica, ha un diametro medio di circa $3.2 \mathrm{~mm}$, mentre nella condizione di angolo pari a $30^{\circ}$ (Fig. 10), l'area di contatto ha forma pseudo-circolare di raggio pari a $3.7 \mathrm{~mm}$. 


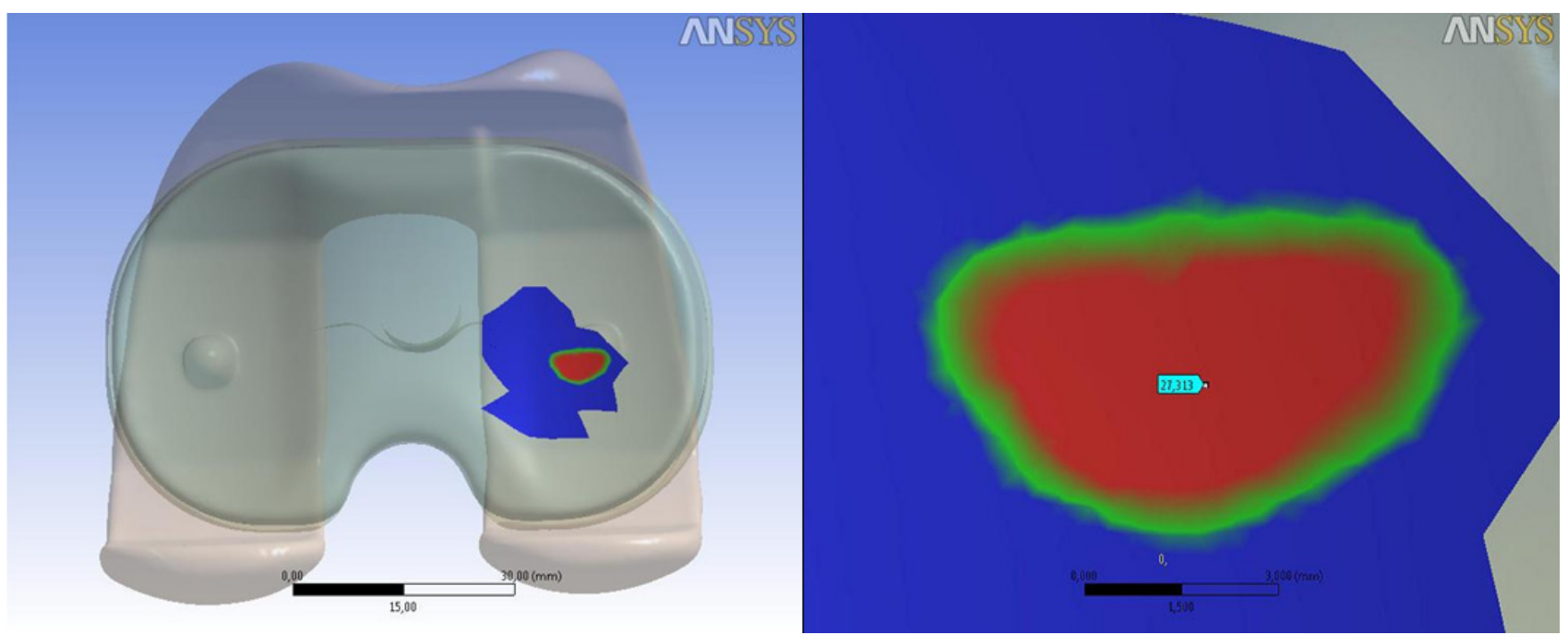

Figura 9: Configurazione a $0^{\circ}$ : area di contatto e pressione massima.

Figure 9: Configuration at $0^{\circ}$ : contact area and maximum pressure.

In entrambi i casi, eventuali piccole irregolarità delle aree di contatto possono essere ricondotte agli inevitabili errori ed al rumore generato durante la fase di acquisizione e ricostruzione della forma. Nei casi analizzati, tuttavia, gli errori risultano di trascurabile entità. Le pressioni massime calcolate sono pari a 27 e $30 \mathrm{MPa}$ rispettivamente per la condizione a $0^{\circ}$ e $30^{\circ}$ e tali valori risultano inferiori al limite di rottura del materiale.
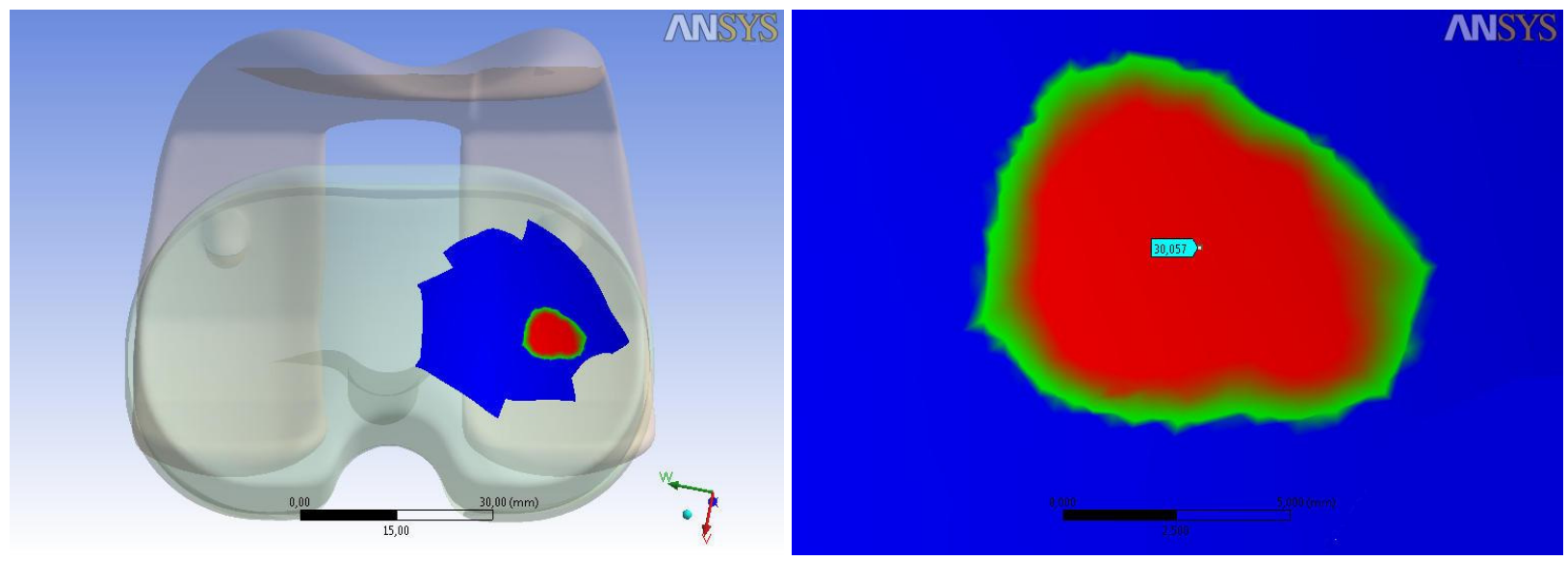

Figura 10: Configurazione a $30^{\circ}$ : area di contatto e pressione massima.

Figure 10: Configuration at $30^{\circ}$ : contact area and maximum pressure.

\section{VALUTAZIONE SPERIMENTALE DELL'AREA DI CONTATTO}

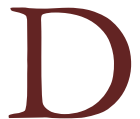

al punto di vista ultrasonoro, il coefficiente di riflessione in corrispondenza dell'interfaccia di separazione tra due mezzi perfettamente aderenti e caratterizzati da un certo valore di impedenza acustica $Z_{1}$ e $Z_{2}$ è espresso dalla relazione $\mathrm{R}=\left(\mathrm{Z}_{2}-\mathrm{Z}_{1}\right) /\left(\mathrm{Z}_{2}+\mathrm{Z}_{1}\right)$. Un incremento della pressione di contatto si traduce in un aumento dell'area reale di contatto e quindi una porzione minore delle onde ultrasoniche verrà riflessa. L'osservazione di una diminuzione dell'ampiezza del segnale riflesso denota uno stato del contatto più intimo.

Questi concetti sono stati applicati per analizzare diversi casi pratici di contatto come l'interazione tra ruota e rotaia ferroviaria, articolazioni umane, giunzioni bullonate. In tutte queste situazioni il metodo ultrasonoro ha mostrato la sua validità nel fornire utili informazioni sull'area nominale di contatto, area reale di contatto e sulla pressione di contatto. 
Esprimendo il coefficiente di riflessione R come rapporto tra l'ampiezza dell'onda incidente $\left(\mathrm{H}_{0}\right)$ in assenza di carico e l'ampiezza dell'onda riflessa per un dato carico applicato $\left(\mathrm{H}_{\mathrm{i}}\right), \mathrm{R}$ assume due valori estremi. $\mathrm{Ha}$ valore unitario quando nessuna quota di energia ultrasonica è trasmessa attraverso l'interfaccia (carico esterno nullo), valore nullo quando tutta l'energia è trasmessa attraverso l'interfaccia, ciò implica che ciascun punto delle due superfici è in perfetto contatto (situazione ideale). In pratica un'interfaccia sollecitata sarà caratterizzata da un valore intermedio del coefficiente di riflessione, valore dipendente dalle condizioni del contatto. Quindi l'analisi quantitativa della riflessione ultrasonora può essere impiegata per definire i confini della regione di contatto mediante l'elaborazione grafica dei dati sulla riflessione, che consente la costruzione di mappe cromatiche in cui si delinea l'estensione e il grado di accoppiamento in funzione della tonalità associata.

I dispositivi utilizzati per eseguire le prove sperimentali sono una macchina prova materiali, un sensore, un pulser, un ricevitore di segnali ed un oscilloscopio. Il sensore utilizzato è di tipo piezoelettrico, ad immersione, con frequenza nominale di $4 \mathrm{MHz}$ e diametro $10 \mathrm{~mm}$. Durante l'esecuzione delle prove è stato necessario ridurre del $50 \%$ il diametro dell'area attiva del cristallo mediante un apposito riduttore, in modo tale da incrementare la risoluzione delle acquisizioni.

Per garantire l'applicazione dell'esatto carico tra i componenti costituenti la protesi in esame, è stato necessario strumentare il provino in modo tale da riuscire ad interfacciarlo con la macchina prova materiali INSTRON, che permette una regolazione precisa del carico imposto. Ciò è stato fatto mediante l'interposizione di dispositivi di ancoraggio, realizzati per permettere sia l'inclinazione relativa che il corretto allineamento con la traversa mobile della macchina. Inoltre nella componente femorale si è creato un involucro di contenimento per l'acqua, in quando le analisi sono state effettuate ad immersione (Fig. 11).

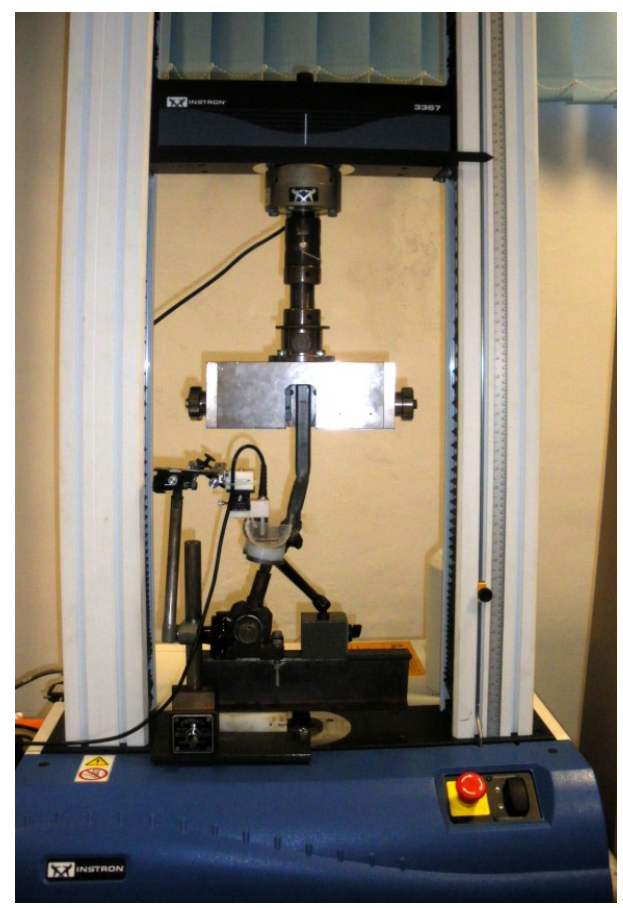

Figura 11: Protesi montata sulla macchina prova materiali.

Figure 11: Knee prosthesis installed in the material testing machine.

La natura visco-elastica dell'UHMWPE è tale da provocare una variazione del livello di carico imposto a causa di un aumento della deformazione. Questo decremento di carico provoca una non corretta valutazione dei risultati. Per epurare le analisi da questo effetto spurio, è stata condotta una prova di carico preliminare in controllo di spostamento per valutare l'andamento del carico in funzione del tempo. Dopo una prima fase in cui il decremento è di tipo esponenziale, la pendenza risulta meno marcata e tale da garantire la corretta valutazione del carico applicato a meno di variazioni pari al $10 \%$; ritenute ininfluenti ai fini dell'analisi. Da ciò scaturisce l'esigenza di effettuare tutte le acquisizioni dopo un periodo iniziale di stabilizzazione pari a $300 \mathrm{~s}$.

Le prove sperimentali sono state precedute da uno studio preliminare, volto a determinare il livello di attenuazione delle onde ultrasonore, dovuto alla diffusione causata dalla particolare finitura superficiale della componente femorale. Le asperità si comportano da minuscoli ostacoli, i quali complessivamente danno origine ad una dispersione dell'energia 
incidente in più direzioni. La quantità di energia diffusa dipende dalle condizioni di rugosità della superficie su cui le onde acustiche incidono, dal valore della lunghezza d'onda e dall'angolo d'incidenza. Normalmente una superficie è considerata speculare (assenza di diffusione) quando la relativa rugosità $\mathrm{R}_{\mathrm{t}}$ è inferiore al 10\% della lunghezza d'onda $(\lambda)$. Per rugosità maggiori il fenomeno della diffusione non è trascurabile ed aumenta al crescere di $\mathrm{R}_{\mathrm{t}}$. La rugosità conferita alla protesi durante il processo produttivo può considerarsi dell'ordine di $60 \mu \mathrm{m}$ [19]. Quindi la lunghezza d'onda dell'onda longitudinale deve essere maggiore di $0.6 \mathrm{~mm}$ per avere assenza di diffusione. Considerato che la velocità dell'onda longitudinale $\left(\mathrm{v}_{\mathrm{L}}\right)$ nel CoCrMo è di $5.88 \mathrm{~mm} / \mu$ s basta utilizzare una sonda con frequenza inferiore ai $9 \mathrm{MHz}\left(\mathrm{v}_{\mathrm{L}}=\lambda \cdot \mathrm{f}\right)$. Con la sonda utilizzata (4 MHz) la lunghezza d'onda è pari a $1.47 \mathrm{~mm}$ (rispettando la relazione $\mathrm{R}_{\mathrm{t}}<0.1 \lambda$ ) e il segnale non subisce un'attenuazione sostanziale nella zona con rugosità (pari al $6.7 \%$ rispetto ad una zona speculare).

La valutazione sperimentale del contatto interessa la superficie all'interfaccia tra la componente femorale e l'inserto tibiale della protesi. I carichi adottati sono, come nel caso delle prove numeriche, di $900 \mathrm{~N}$ per un angolo di inclinazione tra la componente femorale e quella tibiale pari a $0^{\circ}$ e di $2700 \mathrm{~N}$ corrispondenti ad un angolo di $30^{\circ}$.

La metodologia di acquisizione adottata per le prove è il C-scan, che fornisce la rappresentazione bidimensionale dell'estensione dell'area di contatto. Il percorso del sensore durante le analisi è stato guidato mediante uno schema a matrice, attraverso l'adozione di una guida che consente di controllare il movimento lungo due direzioni perpendicolari (Fig. 12). La zona ispezionata ha una dimensione di $15 \mathrm{~mm}$ x $15 \mathrm{~mm}$, con acquisizioni fatte con passo $0.5 \mathrm{~mm} \times 0.5 \mathrm{~mm}$ ottenendo in tal modo una matrice $31 \times 31$ elementi.
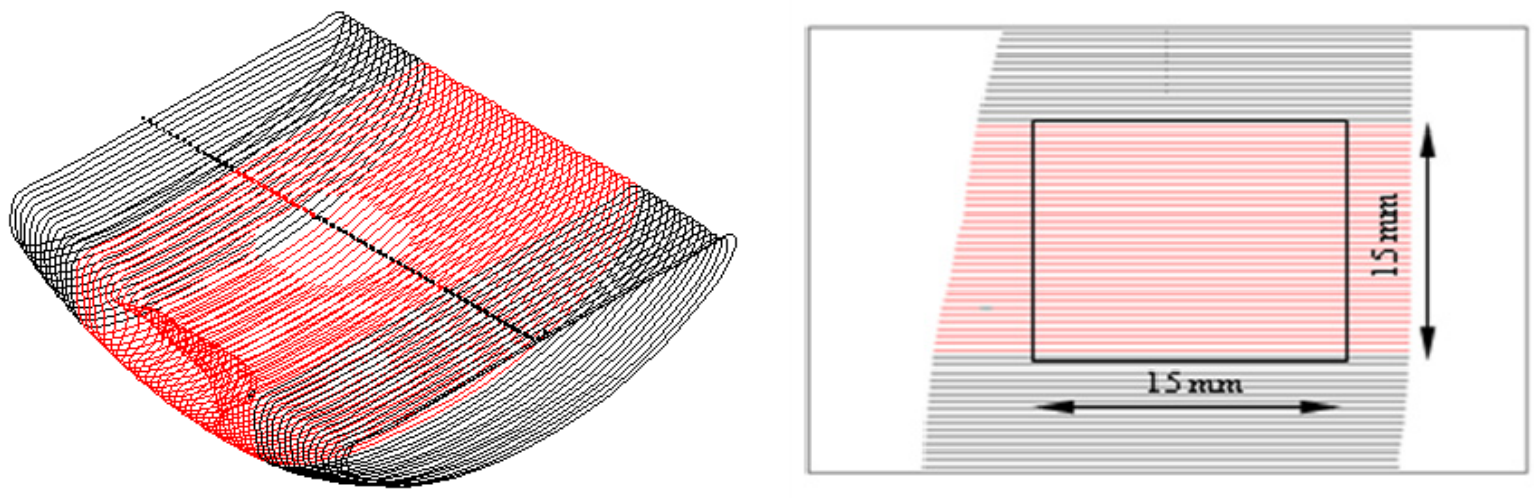

Figura 12: Sezioni realizzate per definire le caratteristiche geometriche dell'area d'interesse.

Figure 12: Sections made to define the geometric characteristics of the UT scan area.

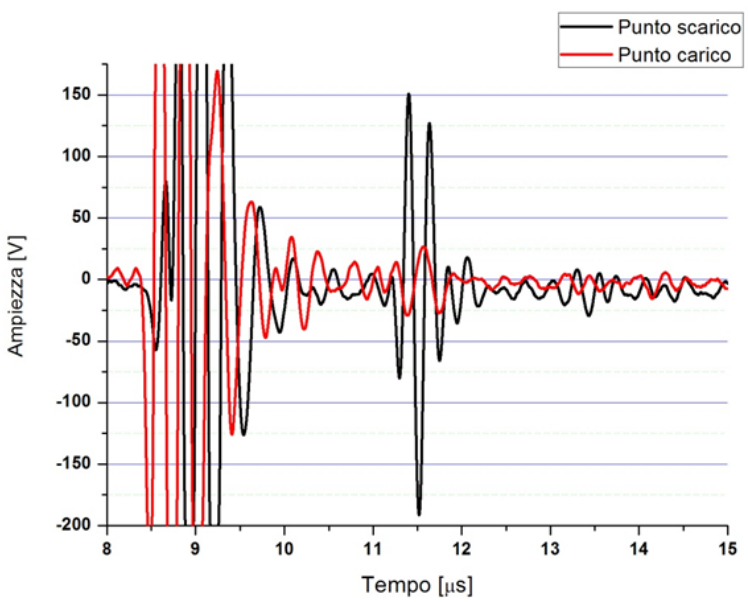

Figura 13: Onda riflessa acquisita in assenza e in presenza di carico.

Figure 13: Reflected wave acquired without (black) and with load (red).

Le mappe cromatiche tracciate riportano il coefficiente di riflessione $\mathrm{R}$ valutato come rapporto di due segnali acquisiti nel medesimo punto con le stesse configurazioni, in presenza e in assenza di carico. La presenza del contatto è tale da diminuire l'ampiezza dell'eco generato all'interfaccia tra i componenti accoppiati (Fig. 13), perché parte dell'energia viene trasmessa al componente sottostante. Tale diminuzione risulta proporzionale al grado di accoppiamento. 
La Fig. 14 mostra le mappe cromatiche che forniscono una chiara definizione dell'estensione del contatto per entrambi i livelli di carico. Per il carico di $900 \mathrm{~N}$ ed angolo $\theta=0^{\circ}$, il raggio medio è di $3.1 \mathrm{~mm}$; per il carico di $2700 \mathrm{~N}$ ed angolo $\theta=30^{\circ}$ il raggio medio è di $3.5 \mathrm{~mm}$.

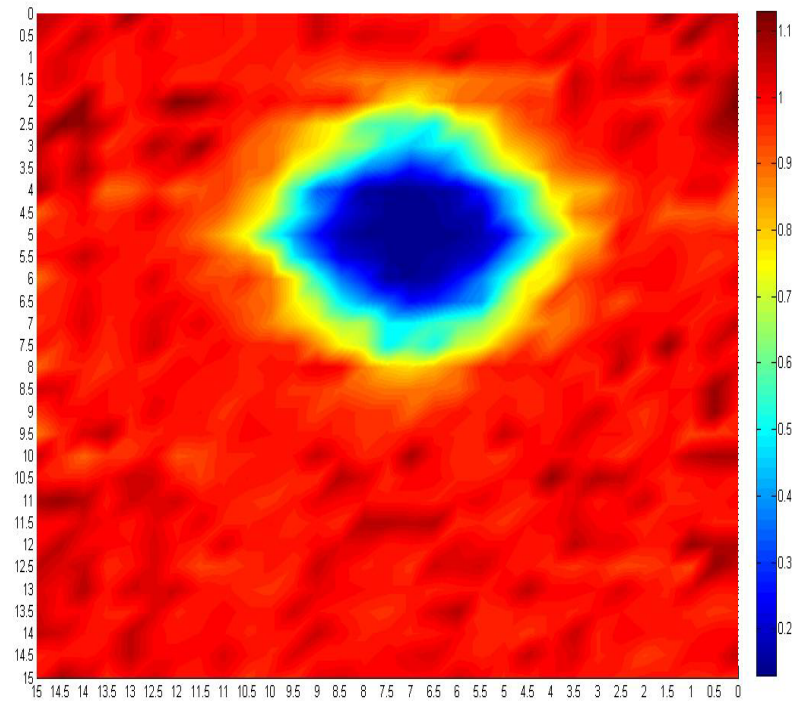

(a)

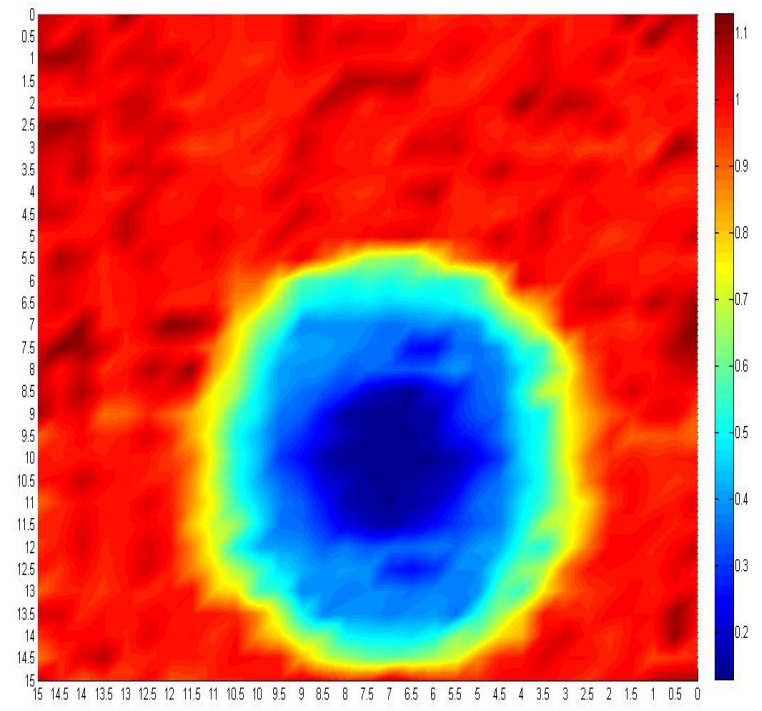

(b)

Figura 14: Mappa cromatica che identifica l'estensione dell'area di contatto relativa ad un carico di $900 \mathrm{~N}$ (a) e di $2700 \mathrm{~N}$ (b).

Figure 14: Color maps identifying the extension of the contact area relative to a load of $900 \mathrm{~N}$ (a) and $2700 \mathrm{~N}$ (b).

Dal confronto delle mappe, come rilevato anche numericamente, è possibile osservare che ad un incremento del carico corrisponde un'area di contatto maggiore ed, al variare dell'angolo $\theta$, il contatto si sposta in accordo al movimento di roto-traslazione a cui la protesi è soggetta durante la normale flesso-estensione dell'articolazione (Fig. 15).

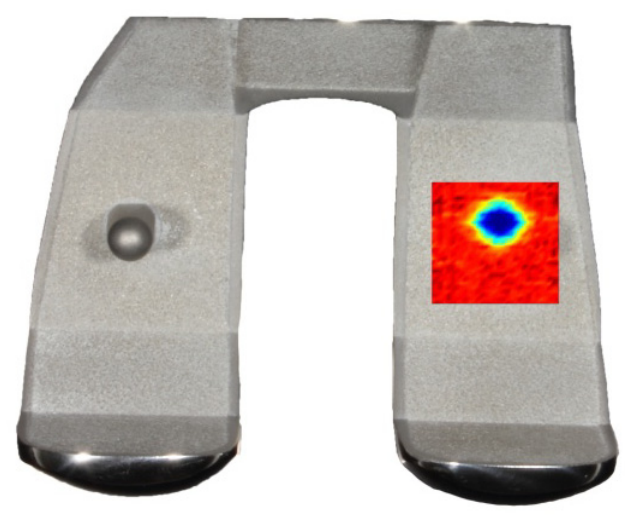

(a)

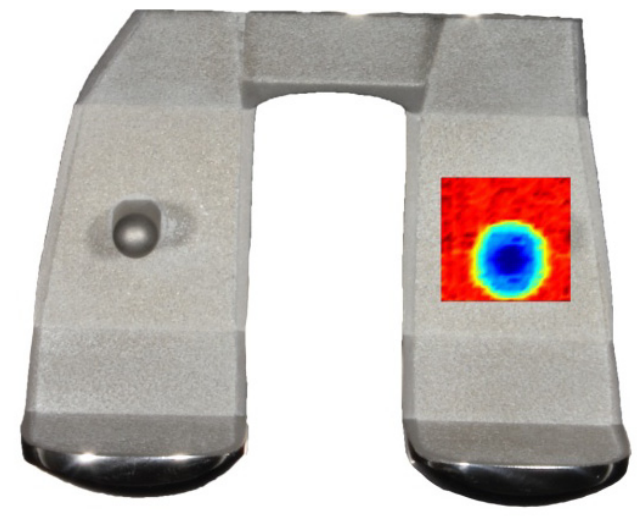

(b)

Figura 15: Posizione relativa dell'area di contatto per il carico di $900 \mathrm{~N}$ (a) e di $2700 \mathrm{~N}$ (b). Figure 15: Relative position of the contact area for $900 \mathrm{~N}$ (a) and $2700 \mathrm{~N}$ (b).

\section{CONFRONTO DEI RISULTATI NUMERICI E SPERIMENTALI}

$\mathrm{P}$

er quanto riguarda il confronto dei risultati ottenuti seguendo i due differenti approcci, è possibile affermare quanto segue. I dati ottenuti con i due metodi sono paragonabili. I risultati sperimentali sono congruenti con quelli trovati per via numerica tramite analisi FEM. 
Come riassunto dalla Tab. 2, il grado di correlazione tra i dati sperimentali e numerici varia da un minimo di $94 \%$, per la condizione a $30^{\circ}$, ad un massimo di $97 \%$ per la condizione a $0^{\circ}$. Di conseguenza è possibile affermare che le procedure utilizzate per la determinazione dell'area di contatto sono entrambe efficaci ed in grado di fornire risultati molto attendibili. La procedura numerica può essere usata per determinare l'area di contatto per diversi angoli e per diversi carichi. Essa, inoltre, ha il vantaggio di poter essere utilizzata durante la fase di progettazione al fine di ottimizzare le geometrie delle superfici, riducendo la pressione al contatto e, conseguentemente, aumentando la vita utile della protesi. La tecnica ad ultrasuoni, invece, può essere efficacemente usata per validare i dati numerici successivamente alla fase di realizzazione del prototipo.

\begin{tabular}{ccccc}
\hline $\begin{array}{c}\text { Forza } \\
\text { applicata }\end{array}$ & Angolo & $\begin{array}{c}\text { Raggio } \\
\text { sperimentale }\end{array}$ & $\begin{array}{c}\text { Raggio } \\
\text { numerico }\end{array}$ & $\begin{array}{c}\text { Grado di } \\
\text { correlazione }\end{array}$ \\
\hline $900 \mathrm{~N}$ & $0^{\circ}$ & $3.1 \mathrm{~mm}$ & $3.2 \mathrm{~mm}$ & $\mathbf{9 7 \%}$ \\
$2700 \mathrm{~N}$ & $30^{\circ}$ & $3.5 \mathrm{~mm}$ & $3.7 \mathrm{~mm}$ & $\mathbf{9 4} \%$ \\
\hline
\end{tabular}

Tabella 2: Grado di correlazione tra i risultati sperimentali e numerici, ottenuti per le due tipologie di carico e $\theta$.

Table 2: Correlation between the experimental and numerical results for the two loading conditions.

Allo stato attuale non è possibile confrontare i dati relativi al calcolo della pressione massima agente all’interfaccia, ottenuti esclusivamente per via numerica. I risultati ottenuti, tuttavia, mostrano un elevato grado di sollecitazione del polietilene che risulta stressato oltre il limite di snervamento ma al di sotto del carico di rottura.

\section{CONCLUSIONI E SVILUPPI FUTURI}

I n questo lavoro è stata eseguita un'indagine sull'area di contatto fra le componenti della protesi Multigen Plus della Lima-Lto. Sono stati utilizzati e confrontati due differenti approcci: numerico e sperimentale. Lo stato del contatto è stato studiato inizialmente dal punto di vista numerico attraverso il metodo agli elementi finiti. La forma della protesi è stata acquisita tramite lo scanner 3D COMET 5 e, successivamente, sono stati realizzati gli assemblati modelli CAD per le due condizioni di carico $\left(0^{\circ}\right.$ e $\left.30^{\circ}\right)$ analizzate. Le condizioni al contorno imposte durante la messa a punto delle simulazioni FEM sono state tali da riprodurre sperimentalmente il setup di carichi e vincoli utilizzato per le prove sperimentali.

Il metodo ad ultrasuoni, basato sulla tecnica pulse-echo, è stato usato per determinare il coefficiente di riflessione R, valutato come rapporto dei due segnali acquisiti nel medesimo punto, in presenza e in assenza di carico.

I risultati ottenuti mostrano un alto livello di correlazione fra l'approccio numerico e quello sperimentale. La procedura numerica, utilizzata nella fase di prototipazione virtuale, permette lo studio di diverse soluzioni in tempi ridotti e con costi contenuti, e può favorire la ricerca di soluzioni ottimali in grado di migliorare le prestazioni e la vita utile delle protesi. Gli ultrasuoni possono, invece, essere utilizzati per validare i dati numerici effettuando le verifiche sui prototipi fisici.

Gli sviluppi futuri riguarderanno lo sviluppo e la validazione di una procedura per la stima della pressione al contatto a partire dai dati sperimentali. Per far ciò si potrà fare ricorso ad un approccio misto teorico/sperimentale, effettuando una correlazione tra la pressione calcolata per via analitica mediante la teoria di Hertz e il livello di riflessione ultrasonora, registrato all'interfaccia del contatto. In questo modo si potrebbe ottenere una curva di calibrazione, necessaria per esprimere il legame tra coefficiente di riflessione e la pressione nominale di contatto, da utilizzare come chiave di lettura delle mappe cromatiche, al fine di determinare i valori di pressione corrispondenti nelle zone interessate al contatto.

\section{BIBLIOGRAFIA}

[1] F. Barney Le Veau, Biomeccanica del movimento umano, Verducci Editore (1993).

[2] J. B. Morrison, J. Biomech., 3 (1970) 51.

[3] T. Ingrassia, L. Nalbone, V. Nigrelli, D. Tumino, V. Ricotta, Int. J. Interactive Design Manufacturing, (2012) 1, DOI: $10.1007 / \mathrm{s} 12008-012-0167-7$.

[4] M.L. Harris, P. Morberg, W.J. Bruce, W.R. Walsh, J. Biomech, 32 (1999) 951.

[5] J. J. Liau, C. C. Hu, C. K. Cheng, C. H. Huang, W. H. Lo, Clin. Biomech., 16(2) (2001) 160. 
[6] J. Krautkrämer, H. Krautkrämer, Ultrasonic Testing of Materials, Springer- Verlag, Berlin (1983).

[7] M. Arone, D. Cerniglia, V. Nigrelli, J. Mater. Process. Technol., 176 (2006) 95.

[8] A. Pantano, D. Cerniglia, Appl. Phys. A: Mater. Sci. Process., 91 (2008) 521.

[9] A. Pantano, D. Cerniglia, Appl. Phys. A: Mater. Sci. Process., 98 (2010) 327.

[10] D. Cerniglia, A. Pantano, C. Mineo, Appl. Phys. A: Mater. Sci. Process., 105 (2011) 959.

[11] S. Kenderian, B.B. Djordjevic, D.Cerniglia, G. Garcia. Insight, 48 (2006) 336.

[12] D. Cerniglia, A. Pantano, M.A. Vento, J. Nondestruct. Eval. , 31 (2012) 245.

[13] http://www.lima.it/products-multigen_plus-1.html

[14] R. Chiesa, M.C. Tanzi, S. Alfonsi, L. Paracchini, M. Moscatelli, A. Cigada, J. Biomedical Mater. Research, 50 (3) (2000) 381.

[15] F. Chen, G.M. Brown, M. Song, Optical Engineering, 39(1) (2000) 10.

[16] T. Ingrassia, F. Cappello, Int. J. Interactive Design and Manufacturing, 3(1) (2009) 1.

[17] J.C. Simo, T.A. Laursen, Computers and Structures, 42 (1992) 97.

[18] A.C. Godest, M. Beaugonin, E. Haug, M. Taylor, P.J. Gregson, J. Biomechanics, 35 (2002) 267.

[19]J. Bellemans J., Acta Orthop Scand Suppl., 288 (1999) 1. 